Archives de sciences sociales des religions

133 | janvier - mars 2006

Catholicismes

\title{
Un religieux immanent et transnational
}

\section{André Corten}

\section{OpenEdition}

Journals

Édition électronique

URL : http://journals.openedition.org/assr/3339

DOI : 10.4000/assr.3339

ISSN : $1777-5825$

Éditeur

Éditions de l'EHESS

Édition imprimée

Date de publication : 1 mars 2006

Pagination : 135-151

ISBN : 2-7132-2046-7

ISSN : 0335-5985

Référence électronique

André Corten, «Un religieux immanent et transnational », Archives de sciences sociales des religions [En ligne], 133 | janvier - mars 2006, mis en ligne le 03 mai 2009, consulté le 01 mai 2019. URL : http:// journals.openedition.org/assr/3339; DOI : 10.4000/assr.3339 


\section{André Corten*}

\section{Un religieux immanent et transnational}

Le pentecôtisme, né au tout début du $\mathrm{XX}^{\mathrm{e}}$ siècle, est un mouvement de renouveau du protestantisme, en particulier du méthodisme et du baptisme. En tant que tels, ses référents théologiques relèvent explicitement de la transcendance. Si le pentecôtisme accorde une importance à la figure de l'Esprit saint, aucun doute n'est formulé sur le caractère divin et transcendant de celui-ci. Cependant, cette figure étant convoquée en termes d'effusion émotionnelle et présentée dans les discours de certaines Églises comme le plus fort des esprits, c'est en fonction des sociétés dans lesquelles le pentecôtisme a du succès que la question de l'immanence se pose. Le développement du pentecôtisme depuis 1980 et l'apparition de ce qu'on appelle le "néo-pentecôtisme » a fait surgir de nouveaux traits relevant de l'immanence : d'une part la centralité de l'argent dans les rituels et dans la gestion des organisations et d'autre part une capacité surprenante à dépasser les frontières nationales et à se soustraire à l'aura de transcendance attachée à la forme de l'État souverain.

Avant de pouvoir traiter de ce religieux pentecôtiste immanent et transnational, il convient de s'interroger sur les catégories d'immanence et de transcendance. Importées de la théologie et de la philosophie, ces catégories sont entrées dans le champ de la sociologie et de l'histoire politique. Mais dans l'essor de théories récentes, subsiste une ambiguïté qui paraît consubstantielle. On pense à des expressions comme " transcendance immanente » de Maffesoli (1999) ou " absolu terrestre » de Gauchet (2004) ${ }^{1}$. Ces ambiguités sont le reflet d'une double évolution, l'évolution du religieux, mais aussi l'évolution du politique dans les sociétés contemporaines. Une des manifestations de cette évolution du politique est la transnationalisation traduisant un certain changement de la forme de l'État. Si tant au plan politique que religieux, on peut observer des tendances à l'immanence, il persiste toujours, ne fût-ce que comme «sursaut éthique », des facteurs de transcendance.

* Professeur de sciences politiques et d'analyse du discours à l'Université du Québec à Montréal. Membre du GRIPAL (Groupe de recherche sur les imaginaires politiques en Amérique latine. www.gripal.ca). Cette étude est subventionnée par le Conseil de recherche en sciences humaines du Canada (CRSH).

1. Ferry, Gauchet, 2004.

ARCHIVES DE SCIENCES SOCIALES DES RELIGIONS 133 (janvier-mars 2006), p. 135-151 
I36 - ARChives DE SCIENCES SOCIALES DES RELIGIONS

\section{Transcendance et immanence}

Des sens donnés à transcendance, deux méritent d'être retenus ici. Le premier sens répond à la notion de "haut ", de " au-dessus " et de supérieur. S'y ajoute la notion de solution de continuité. Une réalité est transcendante par rapport à une autre lorsque la première ne peut être atteinte par la seconde - de niveau moyen - par un simple mouvement continu. Le second sens est à rapporter à Kant : est transcendant ce qui est "au-delà de toute expérience possible, soit en parlant de principes de réalités, d'êtres; soit en parlant de principes de connaissance $»^{2}$.

Pour le premier sens, l'ajout de la solution de continuité apparaît nécessaire pour donner à l'" au-dessus » une force métaphorique de supériorité absolue. L'image du Ciel répond à cette exigence. Le Ciel est d'un autre ordre, il ne se mesure pas en altitude. La première définition rejoint celle du sacré qui implique fondamentalement l'idée d'un autre ordre sans continuité possible entre l'un et l'autre. Dans le second sens, la notion d'expérience sensible ou cognitive, qui paraît à première vue très nette, se trouve débordée par celle d'expérience émotionnelle. L'expression d'expérience mystique implique paradoxalement un rapport continu (d'expérience) avec un ordre discontinu. Transcendant s'accorde avec un certain type de religieux, le religieux monothéiste. Le christianisme répond aux deux sens de transcendant, Dieu est au-dessus de tout et est inaccessible, il échappe à toute expérience sensible et à toute tentative de démonstration rationnelle. Transcendant s'accorde aussi avec une forme politique. Il s'accorde avec la forme de l'État absolutiste inaugurant l'État moderne. Ici contrairement au monothéisme, on ne peut pas dire que l'État échappe à toute expérience de connaissance.

L'immanence est un concept plus complexe. Loin d'être le simple terme contradictoire de la transcendance (c'est-à-dire non-transcendance), loin de pouvoir se fixer sur la réalité visible par opposition à l'invisible comme Aristote s'en est fait le théoricien, l'immanence est un concept polysémique et en même temps instable, comme le relevaient Deleuze et Guattari (1991). Est immanent à un être (ou à un ensemble d'êtres) ce qui ne résulte pas d'une action extérieure. L'immanence peut donc désigner l'immersion totale dans un monde dans lequel visible et invisible ne sont pas distincts, le tout dépendant d'une vérité inaugurale inaccessible pour la conscience individuelle mais néanmoins comprise dans ce monde. Cette immanence est, selon Gauchet (1985), le propre des « religions premières ». Le terme d'immanence peut au contraire désigner l'autonomie la plus complète de l'être. Cela n'implique pas son isolement. L'immanence est, selon Castoriadis (1975, p. 256), l'émergence de l'altérité radicale. Mais en raison de son manque de fondement, l'immanence est vue également par certains comme la dissolution du sens. La démocratie comme gouvernement de gens n'ayant aucune qualité (aucun fondement) pour gouverner est alors vue comme

2. LALANDE, 1968, p. 1144. 
populisme ${ }^{3}$. Mais plus globalement, c'est la démocratie qui, en sapant les bases de réflexivité de l'individu, va, selon Gauchet (2002), contre elle-même.

\section{Pentecôtisme classique et " néo-pentecôtisme "}

Durant de nombreuses décennies, le pentecôtisme occupe la place de la "secte» tentant de se démarquer des autres courants religieux par sa pureté d'interprétation des textes sacrés et par son refus de compromission avec le "monde ». Dans des sociétés en mutation, il est le refuge des masses ${ }^{4}$. Marion Aubrée (1998) y relève des images renvoyant à la transcendance. Aujourd'hui, le pentecôtisme est encore traité de « secte " par l'Église catholique et, souvent, par des autorités publiques. Mais il a changé ; il ne se positionne plus en « retrait du monde ». On distingue à cet effet le "pentecôtisme classique » et le «néopentecôtisme ».

Qu'entend-on par pentecôtisme classique ? Généralement, on désigne par là le mouvement se développant simultanément dans plusieurs pays (États-Unis, Afrique du Sud, Brésil, etc.) à partir de 1900 jusque dans les années 1950 et même 1980 et se structurant notamment dans quelques grandes Églises comme l'Assemblée de Dieu, l'Église de Dieu, l'Église de Dieu en Christ, ou au Brésil, la Congrégation chrétienne, etc. Il touche en particulier des milieux paupérisés d'Amérique latine et d'Afrique (mais aussi d'Amérique du Nord). S'y constituent des néo-communautés ${ }^{5}$ maintenant les croyants en retrait du monde.

Le « néo-pentecôtisme " ${ }^{6}$ s'affiche "dans le monde ». On y relève par rapport au pentecôtisme classique deux traits apparemment contradictoires: un plus grand relativisme et un manichéisme plus prononcé. Le «néo-pentecôtisme » se développe à travers une myriade de petites Églises animées par des pasteurs charismatiques et/ou entrepreneurs ${ }^{7}$. Il prend aussi la forme de méga-Églises. L'Église Universelle du Royaume de Dieu, cette Église brésilienne comptant deux millions de fidèles au Brésil et un autre million répartis dans 80 pays et dont le sigle en portugais est IURD, sera pris ici comme illustration principale ${ }^{8}$. D'un coté, l'Église Universelle manifeste une grande tolérance de fait vis-à-vis de l'évolution des mœurs et une grande adaptation aux nouvelles techniques (notamment de communication) et d'un autre côté s'y affichent de nouvelles formes de manichéisme avec une vision du monde en termes de guerre entre le

3. «Populisme, dit RanCIÈRE (2005, p. 88), est le nom commode sous lequel se dissimule la contradiction exacerbée entre légitimité populaire et légitimité savante ».

4. LaLIVE D'Espinay, 1970.

5. BASTIAN, 1994.

6. Voir à ce sujet le débat dans ASSR, Willaime, dir., 1999.

7. Marshall-Fratani, 2001.

8. Corten, Dozon, Oro, dirs, 2003. 
Bien et le Mal. Loin d'être néanmoins de teneur fondamentaliste ${ }^{9}$, la capacité du discours d'exorcisme de l'IURD à mobiliser des figures des religions d'origine africaine, témoigne d'une maitrise de la mise en spectacle auquel l'individualisme des paupérisés du tiers-monde est sensible ${ }^{10}$. La délivrance ${ }^{11}$ sanctionne le succès de cette mise en spectacle. Il faut ajouter un troisième trait : la promotion faite d'une doctrine appelée « théologie de la prospérité » qui présente le succès financier comme conforme à la volonté divine. Trois voitures, deux maisons, deux entreprises... Ces démonstrations de " bénédiction divine ", mises en scène dans les émissions télévisées, peuvent tromper l'observateur extérieur. En effet, les couches sociales visées ne sont pas des entrepreneurs, mais des milieux paupérisés. Ainsi, le niveau d'éducation des adeptes de l'IURD est en général inférieur à celui des fidèles des autres Églises.

Qu'il se situe en "retrait du monde » ou de plain-pied dans le monde, le pentecôtisme est vu comme une secte. Après un siècle d'existence, il ne semble pas avoir gravi les échelons de respectabilité des Églises définis par Troeltsch (1912), ni non plus s'être sérieusement embourgeoisé comme le dénonçait Niebuhr (1929). Le pentecôtisme s'est transformé comme les sociétés dans lesquelles il croît. Le pentecôtisme classique correspond à une période de première urbanisation où les nouveaux migrants cherchaient des néo-communautés. Avec une seconde période d'urbanisation, sont apparus les médias électroniques, les programmes d'assistance sociale et l'implication des Églises dans la vie politique (ne fût-ce que pour s'assurer de la liberté religieuse). De plus en plus, les Églises s'impliquent à ces trois niveaux. À cet égard, le « néo-pentecôtisme " est simplement en avance dans une évolution générale. Toutes les Églises suivent la même direction; elles « entrent dans le monde " ${ }^{12}$. Ce qui apparaissait à Marion Aubrée (1998) comme signe de transcendance était la trace de « retrait du monde » d'un mouvement religieux dans sa première phase. S'y manifestaient des structures « imaginales » traduisant ce retrait. Même si le "néo-pentecôtisme » reste tout aussi patriarcal (ce qui renverrait plutôt à une image de transcendance), celui-ci fait apparaître de façon tangible les traits d'immanence du pentecôtisme. Ces traits étaient moins développés mais déjà présents dans le "pentecôtisme classique ».

\section{Le pentecôtisme : une implication ordinaire dans la politique}

Malgré les valeurs transcendantes qu'elle peut parfois invoquer, la vie politique actuelle est une sphère auto-réglée. Le monde politique peut subir des changements

9. Voir à ce sujet l'analyse nuancée de FATH (2004) sur le fondamentalisme aux États-Unis.

10. La représentation de la guerre du «Bien» et du «Mal» est différente de celle qu'on peut observer dans le fondamentalisme américain qui, en fonctionnant de plus en plus comme "religion civile ", revêt également un caractère immanent. CORTEN, 2005.

11. MARY, 2002.

12. J'ai argumenté dans ce sens dans mon article "Pentecôtisme et "néo-pentecôtisme" au Brésil ", Archives de Sciences Sociales des Religions, n 105, janvier-mars 1999, p. 163-183. 
radicaux, mais y réapparaissent constamment les mêmes habitudes, les mêmes compromissions, les mêmes tendances au clientélisme, voire à la corruption. Le monde politique présente l'image de l'immanence au sens le plus ordinaire du terme. Dans un autre sens - on le verra en conclusion -, la vie politique est immanente dans la mesure où elle n'a aucun fondement (extérieur à elle-même). L'espace démocratique est un « lieu vide» disait Lefort (1986).

Pendant longtemps, le pentecôtisme s'est maintenu en retrait de la politique qui était considérée comme le " monde » (de perdition) par excellence. Les croyants se complaisaient dans une attitude millénariste d'attente du second retour du Christ. Tout en se désintéressant des aléas politiques, le pentecôtisme adoptait la maxime paulinienne selon laquelle toute autorité est voulue par Dieu. "Que chacun se soumette aux autorités en charge. Car il n'y a point d'autorité qui ne vienne de Dieu, et celles qui existent sont constituées par Dieu. Si bien que celui qui résiste à l'autorité se rebelle contre l'ordre établi par Dieu » Romains, $13: 1-2$. Respect et indifférence, tels étaient les traits du rapport des pentecôtistes à la politique.

À partir des années 1980, l'implication des pentecôtistes dans la politique tant au Chili, qu'au Guatemala ou qu'au Zaïre se signale par son caractère non démocratique : appui à Pinochet, prêche à la télévision du général-président Rios Montt, appui des protestants à Mobutu. Une césure importante est marquée par l'élection en 1986 de 36 évangéliques à la Constituante brésilienne (dont 20 pentecôtistes). Depuis lors, des partis de type évangélique se forment dans plusieurs pays d'Amérique latine: Salvador, Nicaragua, Venezuela, Haïti ${ }^{13}$. Ailleurs, l'élection d'évangéliques ou, au moins, la recherche de l'appui des Églises évangéliques par les candidats se généralisent : Pérou, Costa Rica, Équateur ${ }^{14}$. En Afrique, les Conférences nationales des années 1990, qui marquent l'abandon au moins formel des partis uniques, ouvrent la voie aux influences religieuses. Dans plusieurs pays, que ce soit le Bénin, le Congo-Brazzaville, le Burkina-Faso, la Côte d'Ivoire, le Kenya ou l'Ouganda, sans parler de l'Afrique du Sud ${ }^{15}$, les évangéliques deviennent des acteurs de la scène politique.

Au Brésil ainsi que dans plusieurs pays, l'engagement des pentecôtistes dans la politique est, au début, justifiée par la défense des libertés religieuses. Elle s'explique aussi par la nécessité, pour ces Églises en expansion, d'exercer des pressions dans les couloirs du parlement, en vue de défendre leurs intérêts en matière d'accès aux médias, de développement des œuvres sociales, de construction de temples, parfois d'éducation. Depuis plusieurs années, l'implication des pentecôtistes devient un élément non négligeable du jeu politique général. À nouveau le Brésil fournit un exemple significatif. Aux élections présidentielles de 2002,

13. BASTIAN et al., 1997, p. 25-27.

14. ANDRADE, 2002, p. 18-27.

15. CORTEN, 2003. 
parmi les quatre candidats, l'un est évangélique (Antony Garotihno) et, arrivé en troisième position au premier tour, a obtenu $16 \%$. Par ailleurs, Lula, président élu au second tour, choisit comme vice-président un sénateur d'un parti (le Parti libéral) dont la moitié des députés appartiennent à l'Église universelle du royaume de Dieu (IURD). En août 2005, l'Église Universelle abandonne les oripeaux du Parti libéral (affecté par les scandales de corruption) et crée un nouveau parti, le PMR (Parti Municipalisé Rénovateur), transformé rapidement en Parti Républicain Brésilien (PRB). Le vice-président de la République, José Alencar, a annoncé son ralliement à ce nouveau parti. La Constitution brésilienne interdisant les partis religieux, on s'interroge sur le caractère " laïque » de ce parti, mais les cerveaux politiques de l'IURD ne sont pas dépourvus de ressources manœuvrières.

Pour Ari Pedro Oro (2003), si les pentecôtistes participent aujourd'hui à la vie politique au Brésil, ils prétendent constamment dans leur discours apporter un supplément d'éthique. De façon plus générale, Renato Janine Ribeiro (2003) avait relevé ce constant appel à la transcendance dans une vie politique brésilienne marquée par l'immanence. Oro notait également que dans la vie parlementaire, les élus pentecôtistes se conformaient rapidement aux procédures du système politique. Ainsi, là où les pentecôtistes sont mal implantés, leur discours dénonce le manque d'éthique dans la politique. Par contre, là où ils ont un certain poids, ils ne se démarquent des autres élus que par leurs positions vis-à-vis des « questions morales » : l'avortement ou le mariage gay. Même en matière de bioéthique, ils ne font pas front commun. Par contre, ils continuent à entretenir une vision de guerre spirituelle se déployant à l'échelle mondiale - Israël devenant à cet égard un référent catégorique. Mais contrairement aux évangéliques des États-Unis, cette position ne joue que faiblement dans les options politiques internationales des pays d'Amérique latine.

L'engagement dans la vie politique est en fin de compte homologue au rapport $\mathrm{au}$ " monde ". Dans la première phase de " retrait du monde ", les pentecôtistes se refusent à toute compromission dans l'univers politique de l'immanence. Dans la phase contemporaine, de la même façon qu'on prend pied dans le «monde ", on s'engage dans la vie politique. C'est vrai pour les Églises pentecôtistes classiques de même que pour les Églises «néo-pentecôtistes ». Les Églises pentecôtistes sont porteuses d'un besoin de supplément d'éthique et de transcendance, au même titre que d'autres instances de la société, effrayées par le nivellement du marché et du relativisme moral ${ }^{16}$.

\section{Imaginaire social de la transnationalisation}

"L'imaginaire social est, primordialement, création de significations imaginaires sociales et création des images ou figures qui en sont le support ", disait

16. Voir RibEIRO, 2003. 
Castoriadis (1975, p. 351). La société instituante se crée à partir de l'imaginaire social. L'État-nation a été créé à partir de figures et d'images, notamment celle de loi et de frontière. À la différence de l'Empire (qui a une vocation universelle), l'État-nation se construit à partir de l'imaginaire social d'une souveraineté fixée spatialement. Elle est pouvoir sur soi-même et la nation délimite le corps sur lequel elle a prise, le distinguant de l'Autre par une frontière et niant toute autorité à la loi de l'Autre. Dans un premier temps, le caractère de droit divin du souverain lui permet de s'émanciper de la tutelle de l'Église ${ }^{17}$. Par la suite, le peuple est lui-même élevé en figure souveraine et acquiert une valeur transcendante. Une certaine symétrie s'établit entre la construction de l'État-nation et celle de l'individu. Selon Gauchet (1985), l'individu se construit comme un corollaire de la transcendance marquant la formation de l'État. Le sujet divin fait apparaitre le sujet humain ouvrant la voie, au plan politique, à la démocratie. Avec l'approfondissement d'un individualisme ordonné par le marché, cette démocratie va se tourner contre elle-même. "La politique de l'individu au présent entraîne une désintellectualisation du fonctionnement social ${ }^{18}$. La société a de moins en moins de saisie sur elle-même.

Mais dans les sociétés du Sud, l'estompage de l'État-nation ne provient pas de cette " politique de l'individu ». L'échec de ce qu'on appelle la modernisation dans le cadre de l'État national résulte du "sous-développement ». Au-delà de ces blocages, cette évolution rend compte d'un autre type de construction du soi ${ }^{19}$. Celui-ci s'inscrit et produit le nouvel imaginaire social de la transnationalisation. Celui-ci est, dans le triomphe de la "mondialisation ", investi de significations imaginaires économiques. Mais dans la «construction de soi » qui s'articule désormais autrement, des renvois de significations s'opèrent et confèrent à la transnationalisation d'autres significations. $\mathrm{Y}$ apparaissent de nouvelles figures et de nouvelles images ouvrant d'autres champs. Celles-ci prennent forme dans des rituels, des mythes et des icônes. On le verra dans la dernière partie de ce texte.

Comme le rappellent Van Dijk (2001, p. 224) ainsi que Marshall-Fratani et Péclard (2002, p. 12), reprenant Foucault, "c'est autant par le recours à ce qu'il appelle des "techniques de soi", dans un "travail de soi sur soi", que selon les codes moraux et des obligations de soumission en vigueur dans une société que l'individu se construit en tant que sujet moral ». Et c'est particulièrement vrai dans le cadre de la transnationalisation. Celle-ci fait des mouvements religieux non pas d'abord un vecteur d'éthique, mais un instrument de " construction du soi ». Le pasteur des nouvelles Églises construit une nouvelle image de soi ; les croyants vont s'y mesurer sans la considérer comme supérieure. Simplement, le pasteur réussit.

17. MANENT, 1987.

18. Gauchet, 2002, p. 368.

19. VAN DIJK, 2001. 
Malgré son apparent conformisme, le pentecôtisme fournit une image inédite de construction de soi et projette celle-ci dans des relations débordant les figures de la loi et de la frontière. De nouveaux espaces se forment, des proximités inattendues apparaissent, des initiatives se libèrent. La conversion individuelle est une "construction du soi » qui prend sa place dans une lutte spirituelle à l'échelle mondiale. Cet imaginaire existe déjà dans la forme traditionnelle du millénarisme qui est une projection tant dans le temps que dans l'espace; c'est cependant la « sécularisation » du millénarisme qui caractérise le développement du pentecôtisme à partir des années 1980. Les grandes croisades, les réseaux inter-dénominationnels, la circulation de vidéos et d'audio-cassettes, les voyages de missionnaires traversant les frontières, l'ouverture de nombreuses succursales d'Églises du Sud en Europe et en Amérique du Nord, voire au Japon, la diffusion à l'échelle mondiale d'émissions télévisées et radiodiffusées, les pèlerinages en Israël ont un caractère pratique d'organisation auquel correspondent aussi des flux financiers. Comme Colonomos (1999) le souligne, ces flux ne vont pas nécessairement du Nord vers le Sud. Ils donnent lieu au développement d'une structure néo-clientéliste se caractérisant par ses articulations plus horizontales que verticales. Les dénominations pentecôtistes et néo-pentecôtistes s'y mêlent. Certaines sont très peu centralisées et fonctionnent selon un système de franchise. On reprend par exemple le nom d'Assemblée de Dieu auquel on ajoute un qualificatif; se développent de façon à la fois homologue et parallèle une multitude de petites ou moyennes Églises qui empruntent aussi des traits aux nouvelles Églises transnationales ou nationales émergentes. Des méga-Églises comme RHEMA (fondée en 1978 par le célèbre théologien de la guérison divine et de la prospérité américain, Kenneth Hagin) prennent parfois des formes tout à fait locales : au Congo et au Rwanda $^{20}$. Il en est de même par exemple des Assemblées de Dieu au Burkina Faso $^{21}$.

Les réseaux traversent les frontières. En ce sens, ils sont très différents des formes d'association telles qu'on croit les reconnaître au niveau national et qui font parler de "société civile». Il s'agit de réseaux ne fonctionnant pas sur la base de complémentarité d'intérêts. L'objectif est en quelque sorte immatériel : s'étendre toujours plus. Non seulement par une sorte de volonté de toute puissance - qu'on trouve parfois dans le vocabulaire de l'Église universelle du royaume de Dieu -, mais dans une conviction plus intime liée à la conversion et qui fait que chacun se considère porteur d'une force de transformation qu'il doit communiquer. Ces réseaux qui prennent l'apparence de rapports d'affaires sont ponctués de rituels, souvent déroutants pour ceux qui sont habitués à une tradition axée sur le recueillement.

Une symétrie nouvelle s'établit entre la construction d'un individu qui n'est pas d'abord réflexivité, mais communication ponctuelle dans l'émotion, et la

20. CORTEN, 2001.

21. LAURENT, 2003. 
transnationalisation investie de sens constitutifs d'identités non-territoriales (et notamment d'appartenance à des Églises). La symétrie décrite par Gauchet (1985) était articulée à une image de la transcendance. La nouvelle symétrie est articulée à un principe d'immanence. Le pentecôtisme, et plus largement les mouvements charismatiques $^{22}$ qui lui sont apparentés et regroupent pas moins d'un demi milliard de croyants de par le monde ${ }^{23}$, ne sont qu'un véhicule de sens pour instituer un nouvel imaginaire de la transnationalisation, mais ils obligent à considérer la transnationalisation autrement que comme seul renvoi de significations économiques ${ }^{24}$. De multiples figures et images instituent une société qui n'est pas simplement définie en termes de manque. La société n'est pas fixée comme manque de transcendance. Que l'on considère, à la manière de l'idéologie néolibérale, les inégalités et les exclusions comme des conséquences inévitables de la «mondialisation » ou au contraire qu'on les dénonce, à la manière de l'altermondialisme, les analyses courantes ne voient dans l'immanence que le jeu de la main invisible. Au contraire de ces analyses, on avance ici l'hypothèse que l'individualisme qui se généralise dans le tiers-monde, en particulier dans les milieux paupérisés, et son symétrique - la transnationalisation - ne sont pas des formes vides de sens, ils sont un imaginaire social instituant les bribes d'une société échappant à la figure de la souveraineté.

\section{L'argent comme imaginaire social}

L'argent est l'unité centrale de l'imaginaire économique marchand. De la même façon que la transnationalisation ouvre à des renvois de significations qui ne sont ni simple démarcage de la figure de souveraineté de l'État nation, ni ne sont absorbés par l'imaginaire économique de la mondialisation, l'argent est l'instance immédiate des relations les plus ordinaires auxquelles la nouvelle construction de soi des paupérisés donne, à travers des figures et des images, un sens, y compris religieux. L'imagination confère à ces relations ordinaires un pouvoir instituant et c'est dans ce pouvoir instituant que le pentecôtisme acquiert sa force d'expansion immanente. L'argent prend dans ce nouveau religieux un caractère paradoxal ${ }^{25}$. Il est à la fois sacrifice et défi. Sacrifice, l'argent donné doit être une privation non seulement d'un besoin, mais du nécessaire (de biens absolument nécessaires); il devient l'expression de la dépendance totale vis-à-vis de Dieu. Défi, l'argent donné commande à Dieu de faire sa part: de rendre l'offrande par une pluie de bénédictions. Dans ce jeu de hasard, s'établit un rapport immanent au divin. Ce rapport s'établit dans le sacrifice/ défi des croyants. Il trouve une reconnaissance à travers le succès financier des pasteurs et des

22. POEWe, 1994.

23. FATH, 2004.

24. Voir à ce sujet la discussion avec ApPADURAI entreprise dans l'introduction de CORTEN, Marshall-Fratani, 2001, p. 1-21.

25. Campos, 1997, Marshall-Fratani, 2001. 
Églises qui transmet aux croyants le message suivant: «c'est bien, vous avez osé, vous voyez comment on peut réussir ». Le rôle de valeur d'échange anonyme est subverti pour donner l'occasion de se dépasser et ce dépassement est alors reconnu comme la preuve de celui qui fait le bien. La transaction s'opère sur la base de la conviction proclamée par le croyant d'avoir des droits - on appelle cette foi la « confession positive ». Au lieu d'être une mécanique effroyable écrasant les marginaux, l'argent est dès lors ce qui, grâce à la foi, donne la capacité de prendre ce à quoi on a droit. Au-delà de son aspect monétaire, l'argent donne aux croyants une valeur modale, une force de pouvoir-faire et ou de vouloirfaire, en tout cas une confiance en soi-même. Certes, il y a toujours suspicion de charlatanerie et le caractère scandaleux de la manipulation de l'argent par les Églises désigne l'intervention toujours possible d'un anti-sujet (qui n'attend que le moment de prendre le croyant comme victime). Mais cet anti-sujet est l'enjeu d'un autre rapport à l'argent. Sans risque, pas de preuve de foi. L'Église manipule la croyance selon laquelle seul le croyant peut exiger de Dieu, mais elle donne en même temps un accès au sacré.

"L'impie ne peut rien exiger de Dieu. Le chrétien, s'il ne veut pas donner prise au diable, a le devoir de réclamer ses droits bibliques. Pour ce faire, il a besoin de confesser sa foi, c'est-à-dire déclarer, décréter, établir la bénédiction à laquelle il a droit ou qu'il désire. Confesser n'a donc rien à voir avec le fait de déclarer des péchés ni de supplier Dieu ou d'implorer sa grâce; il s'agit plutôt de proclamer avec une profonde conviction la propriété des droits, exclusifs aux chrétiens, annoncés dans la Bible. Il s'agit donc d'exiger et de déterminer que Dieu, au nom de Jésus-Christ, a accompli ce qu'il a promis à ses brebis fidèles ; la victoire sur le diable, la santé, la prospérité matérielle, une vie d'abondance, triomphante et heureuse ${ }^{26}$.

La doctrine de la "théologie de la prospérité » n'est pas seulement répandue dans le «néo-pentecôtisme ». Elle est diffusée dans de puissantes associations para-ecclésiales implantées dans une centaine de pays. C'est notamment le cas de la Fraternité Internationale des Hommes d'Affaires du Plein Évangile fondée en 1953 aux États-Unis et à un moment proche des Assemblées de Dieu. C'est aussi le cas de confréries comme les Athlètes du Christ. Plus encore que des vecteurs de diffusion, ces associations sont des figures qui permettent à l'imaginaire social de travailler autrement la figure du pauvre.

En Amérique latine, la théologie de la prospérité vient défaire l'imaginaire de la théologie de la libération. Pour cette dernière, le pauvre est une figure transcendante ${ }^{27}$, avec la théologie de la prospérité, le pauvre est celui qui n'a pas osé, qui n'a pas, au risque de tout perdre, lancé le défi à Dieu. Il n'a pas revendiqué ses droits et dans ses rapports à l'argent, il a démissionné. Il n'est pas pour autant coupable et redevable à un principe extérieur; la maladie, le

26. Mariano, 2003, p. 201.

27. Casaldáliga in Corten, 1990, p. 99-122. 
chômage, les problèmes familiaux résultent de forces intérieures à ce monde et dont il faut se libérer. La non-culpabilité doit être assumée : la délivrance des forces du mal ne relève pas d'un exercice spirituel mais d'un " déchargement " (descarrego) d'énergie maligne. D'où dans ce contexte, l'importance du défilé des images de prospérité diffusées à la télévision ou de garants comme ces membres d'associations para-ecclésiales composées de laïcs. Ils viennent témoigner de l'immanence des choses. L'argent sert de point de croisement pour une multitude de narrations circulant dans la vie quotidienne. La banalisation même des récits de miracle devient un mode d'entrée dans tous les pores de la "vie ordinaire ». Le miracle n'est plus seulement guérison de maladie, mais sortie du chômage. La banalisation du miracle est même une adaptation aux règles du marché, mais elle distribue également des valeurs modales (de pouvoir-faire et de vouloir-faire) permettant de donner aux choses les plus ordinaires d'autres significations. Le pentecôtisme transforme ainsi la "mondialisation » en même temps qu'il adapte mentalement les paupérisés aux règles du marché. C'est cette capacité de transformation immanente qui rend compte de son immense succès.

\section{Rituels, mythes et icônes}

Les significations imaginaires sociales et leur activité par renvois à des réseaux inédits de sens se servent de supports. Dans le champ religieux, ces supports sont notamment les rituels, les mythes et les icônes. Dans le pentecôtisme et en particulier le "néo-pentecôtisme ", chacune de ces trois marques confèrent au religieux sa forme immanente. Les rituels donnent au temps et à l'espace leur dimension de religieux; ils impriment un ordre spatial et temporel ${ }^{28}$. Les rituels pentecôtistes sont étonnamment semblables à travers le monde : partout ils aplatissent le hiératique et déplacent le sacré vers l'immanence. Les mythes relient à une origine ou à une fin. Le pentecôtisme ne joue pas à la pureté originelle et son discours ne relève pas d'une «deixis fondatrice ${ }^{29}$. Au contraire, le glissement dans le temps est projeté vers l'avenir et tend à tout recouvrir par une sorte d'effet de débordement. Quant aux icônes qui ont à la fois un aspect de redondance - de copie - et une puissance symbolique, le pentecôtisme leur attribue un rôle ambivalent: on critique les icônes dans leur aspect transcendant, tout en les réincorporant dans l'immanence.

Tout rituel religieux est sans doute un rapport au corps. Dans le pentecôtisme se manifeste un rapport au corps qui, malgré un discours de contrôle sexuel, est de grande expressivité, voire de sensualité. Le corps tout entier est mobilisé dans les cultes pentecôtistes - les chants sont dansés - et les temples offrent parfois le spectacle d'immenses ballets. Les chants eux-mêmes, à l'origine de tradition

28. Cesar, Shaull, 1999.

29. Même la référence au récit de la pentecôte et la pratique de la glossolalie tendent à perdre de leur importance. 
méthodiste, sont dans plusieurs églises des mélodies à la mode sur lesquelles ont été plaquées des paroles de louange. Toute une industrie gospel s'est d'ailleurs développée, mais les groupes musicaux fleurissent aussi à la base, en particulier chez les jeunes. C'est une manière de les attirer dans ces nouveaux mouvements religieux. À travers cette industrie, à travers ces mobilisations, à travers ces lieux de rassemblement, des connivences s'établissent à l'intérieur ou entre tranches d'âges, entre femmes, entre catégories urbaines, parfois entre migrants d'une même origine, par différence avec d'autres groupes. Des frontières se redessinent et se superposent; elles ne répondent pas à une logique d'en haut.

Dans la diffusion du pentecôtisme à l'échelle mondiale on relève de plus en plus une sorte de moteur à deux temps : rituels et marché. C'est ce moteur qui fait se répandre à l'échelle mondiale des archétypes, voire des fables, dont l'effet suscite des interrogations. La deuxième figure d'immanence est en effet produite par le renversement des mythes. Il n'est plus question d'une simple version du millénarisme qui exigerait des croyants d'organiser leur vie en fonction d'un retour imminent du Christ. Ce puissant mythe a été lui-même transformé et s'hybride parfois avec une idéologie de la «fin de l'histoire ». Il ne s'agit plus non plus d'une simple résurgence du «dispensationalisme ». On se souvient de cette doctrine fondamentaliste apparaissant en Angleterre au milieu du XIX ${ }^{\mathrm{e}}$ siècle et surtout aux États-Unis au début du Xx $\mathrm{X}^{\mathrm{e}}$ siècle et remise à la mode par Reagan et selon laquelle l'histoire est divisée en sept périodes. La fable ici prend un caractère plus immanent. C'est du succès même de la transnationalisation du pentecôtisme et de l'expansion incroyable de ses réseaux que se nourrit la croyance dans un changement radical, vécu, au niveau individuel, à travers l'expérience «fabuleuse » de la conversion. Le discours pentecôtiste - et surtout " néopentecôtiste " - est un discours de narration à succès et le succès même de son expansion tend à justifier la valeur des "fables " qu'il véhicule. On relève dans le fonctionnement du mythe un aspect d'immanence qui transforme la nature même du mythe. Au lieu de reposer sur une scène fondatrice (et sur une parole révélée), le mythe fonctionne par le succès de sa diffusion. Au lieu d'instituer des lieux, il se fond dans une toile totale.

Le rapport aux icônes repose en grande partie sur des mythes de représentation. Le transcendant est un principe qui dans plusieurs religions ou confessions fonde l'irreprésentable. Malgré cela, le catholicisme a développé dans le rapport aux images et aux icônes une position conditionnant toute la culture occidentale, et latino-américaine en particulier. L'icône est une instance du sacré. C'est pourquoi aussi, pour marquer leur différence, le protestantisme et particulièrement le pentecôtisme accusent les catholiques d'idolâtrie. Quel est au juste l'enjeu de cette polémique ? Examinons-le à la lumière d'un célèbre épisode de la vie publique brésilienne. " $\mathrm{O}$ chute da santa ${ }^{30}$, incident provoqué par un évêque de l'IURD

30. Le 12 octobre 1995, dans le cadre de l'émission Despertar da Fé de TV Record, l'évêque Sergio Von Helde prétend par ce geste démontrer le caractère non sacré des images. Vu comme 
bousculant une statue de la Nossa Senhora Aparecida, lors du jour de la fête patronale du Brésil, a été considéré comme une erreur tactique importante du point de vue de l'évêque Macedo (dirigeant suprême de l'Église). L'incident est cependant significatif d'une lutte non pour abolir le rapport aux icônes, mais pour le transformer. La " chute da santa » est une tentative maladroite de torsion du champ imaginaire. En fait, il s'agit d'instituer un rapport différent dans l'ensemble des rituels des Églises pentecôtistes - rituels parce que non traditionnels et au contraire en quelque sorte forgés sur le tas - en aplatissant, chez l'autre (catholique), le rapport aux icônes. De même l'utilisation des objets symboliques dans les rituels du culte, comme le fait de façon inventive et dans un continuel souci de renouvellement l'Église universelle, ne doit pas être vue comme le signe d'un rapprochement avec l'Église catholique comme le pensait Sanchis (1992), mais au contraire doit être analysée comme un épisode dans la stratégie de torsion du champ imaginaire en vue de poser un nouvel instituant. Ces objets chaque fois renouvelés entrent une ronde formant la maille d'un nouvel espace : entre le temple et la maison, entre les différents temples, entre les pays.

\section{Conclusion}

Les religions ont presque toujours un caractère transnational. Le christianisme, depuis ses débuts, est transnational et a même contraint l'empire romain à se diviser en deux. Malgré toute l'admiration que saint Augustin porte à l'organisation de l'empire romain, l'Église catholique profite finalement de la chute de celui-ci et devient la seule puissance à prétention d'universalité. La naissance de l'État moderne tient au fait que sans vouloir concurrencer l'Église, le principe de souveraineté parvient, avec l'absolutisme, à se fonder sur un territoire ample mais néanmoins limité. L'État moderne est marqué par cette manière dont a été résolu le problème théologico-politique ${ }^{31}$. Et c'est dans cette perspective qu'il faut penser le rapport du pentecôtisme avec l'État. La séparation Église/État n'est pas transposable à celui-ci. Il n'est pas conditionné par l'acte constitutif de l'État moderne ; en même temps, il tient sa réalité même de son développement transnational.

Le pentecôtisme contient peut-être un autre problème théologico-politique. Le politique avait été conçu comme de l'ordre de la transcendance à partir du principe de souveraineté de l'absolutisme. C'est par réaction aux prétentions de l'Église catholique qu'il l'était devenu. Avec ses traits d'immanence imbriqués dans leur caractère transnational et son nouveau rapport à l'argent, le pentecôtisme est peut-être amené à ébaucher un autre type de politique. La participation à la vie politique du pentecôtisme n'est pas en soi un facteur d'immanence. En général

une provocation non seulement par les catholiques, mais par les milieux cultivés brésiliens, le geste va être rediffusé incessamment sur la grande chaîne télévisée Rede Globo, pour en stigmatiser le caractère scandaleux.

31. MANENT, 1987, p. 17-30. 
d'ailleurs, la sortie du pentecôtisme de sa position de « retrait du monde » n'est pas de façon décisive un passage à l'immanence. À ce titre la distinction entre pentecôtisme et " néo-pentecôtisme » n'est pas radicale. Mais une nouvelle question se pose. Au-delà de son appel à des principes éthiques (à consonance de transcendance), le type d'immanence qui se consolide avec l'expansion du pentecôtisme et des mouvements charismatiques n'est-il pas de nature à conférer au politique un caractère nouveau ? Il viserait en quelque sorte à fonder une immanence qui y est installée. L'État moderne était né du fait de l'incapacité de la cité et de l'empire à résoudre le problème théologico-politique ${ }^{32}$. À quelle forme politique peut donner naissance le nouveau problème théologico-politique lié à l'immanence et à l'individualisme ? Voilà reposée aussi la traditionnelle question de savoir si le pentecôtisme favorise ou est un obstacle à la démocratie ${ }^{33}$. Mais penser le concept de démocratie en termes d'immanence n'oblige-t-il pas à renoncer à toute recherche de fondement ? ${ }^{34}$

André CORTEN

Université du Québec à Montréal (UQÀM)

32. Ibid.

33. GIFFORD, 1995.

34. RANCIÈRE, 1995 et 2005 . Notons que Rancière ne se revendique pas pour autant de l'immanence. 


\section{Bibliographie}

Andrade Susana, "Le réveil politique des Indiens protestants de l'Équateur ", Social Compass, 49/1, mars 2002, p. 13-27.

ARCHIVES DE SCIENCES SOCIALES DES RELIGIONS, Le pentecôtisme : les paradoxes d'une religion transnationale de l'émotion, coordonnateur Willaime Jean-Paul, 44 e année (105), janvier-mars 1999.

ApPAdurai Arjun, Après le colonialisme: les conséquences culturelles de la globalisation, Paris, Petite bibliothèque Payot, 2005.

Aubrée Marion, "La vision de la femme dans l'imaginaire pentecôtiste ", Cahiers du Brésil contemporain, 1998, $\mathrm{n}^{\circ} 35-36$, p. 231-245.

BASTIAN Jean-Pierre, Le protestantisme en Amérique latine : une approche socio-historique, Genève, Labor et Fides, 1994.

BASTIAN Jean-Pierre et al., "La diversification du religieux en Amérique latine : à propos de l'expansion des pentecôtismes ", Problèmes d'Amérique latine, $\mathrm{n}^{\circ} 24$, janvier-mars 1997.

CARrIER Michel, Penser le sacré : les sciences humaines et l'invention du sacré, Montréal, Liber, 2005.

CAMpos Leonildo Silveiro, Teatro, templo e mercado: uma análise da organização, rituais e eficácia comunicativa de um empreendimento neopentecostal: Igreja Universal do Reino de Deus, Petrópolis/São Bernardo do Campos, Vozes/IMES, 1997.

Casaldáliga Pedro Dom, "Entrevue avec Dom Pedro Casaldáliga » in André Corten, Les peuples de Dieu et de la forêt, Montréal-Paris, VLB/L'Harmattan, 1990.

CAstoriadis Cornelius, L'institution imaginaire de la société, Paris, Seuil, 1975.

Cesar Waldo, Shaull Richard, Pentecostalismo e futuro das igrejas cristãs: Promessas e Desafios, Petrópolis/São Leopoldo, Editora Vozes/Sinodal, 1999.

Colonomos Ariel, «Du paria au parvenu : les dimensions internationales du passage au politique ", Ibero-Amerikanisches Archiv, Jahrgang 25, 1999, Heft 1-2, p. 191-210.

Corten André, Les peuples de Dieu et de la forêt, Montréal-Paris, VLB/L'Harmattan, 1990.

Corten André, "Pentecôtisme et "néo-pentecôtisme" au Brésil ", Archives de sciences sociales des religions, $\mathrm{n}^{\circ}$ 105, (1) 1999, janvier-mars, p. 163-183.

Corten André, "Le discours de la réconciliation et les nouvelles Églises au Rwanda ", Afrique contemporaine, $\mathrm{n}^{\mathrm{o}} 200,4^{\mathrm{e}}$ trimestre 2001, p. 65-81.

Corten André, Marshall-Fratani Ruth, dirs, Between Babel and Pentecost. Transnational Pentecostalism in Africa and Latin America, Londres-Bloomington, IN, Hurst Publisher/ Indiana University Press, 2001.

CORTEN André, MARY André, dirs, Imaginaires politiques et pentecôtismes: Afriquel Amérique latine, Paris, Karthala, 2001.

Corten André, «Afrique du Sud : les pentecôtismes dans le recodage du politique par le religieux », Religiologiques, 26, printemps 2003, p. 257-280.

Corten André, "Précarité constitutive de la "transcendance politique" en Amérique latine ", $27^{e}$ Congrès SISR, Turin, 2003.

Corten André, Dozon Jean-Pierre, Oro Ari Pedro, dirs, Les nouveaux conquérants de la foi : L'Église universelle du royaume de Dieu (Brésil), Paris, Karthala, 2003.

Corten André, "Le mal existe" : religion et néo-conservatisme dans le discours de Bush ", Mots, Les langages du politique, $\mathrm{n}^{\circ}$ 69, novembre 2005, p. 9-20.

Deleuze Gilles, Guattari Félix, Qu'est-ce que la philosophie ?, Paris, Minuit, 1991. 
I 50 - ARCHIVES DE SCIENCES SOCIALES DES RELIGIONS

FATH Sébastien, Militants de la Bible aux États-Unis : Évangéliques et fondamentalistes du Sud, Paris, Autrement, 2004.

Ferry Luc, Gauchet Marcel, Le religieux après la religion, Paris, Nouveau collège de philosophie/Grasset, 2004.

GAUCHET Marcel, Le désenchantement du monde : Une histoire politique de la religion, Paris, Gallimard, 1985.

Gauchet Marcel, La démocratie contre elle-même, Paris, Gallimard (coll. «Tel »), 2002.

GIFFORD Paul, dir., The Christian Churches and the Democratization of Africa, Leyde, Brill, 1995.

LALANDE André, Vocabulaire technique et critique de la philosophie, Paris, PUF, 1968.

Lalive D’Espinay Christian, El Refugio de las Masas, Santiago de Chile, Ed. Pacífico, 1970.

Laurent Pierre-Joseph, Les pentecôtistes au Burkina Faso. Mariage, pouvoir et guérison, Paris, IRD-Karthala, 2003.

LEFORT Claude, " Permanence du théologico-politique », Essais sur le politique, Paris, Seuil, 1986, p. 251-300.

Maffesoli Michel, La violence totalitaire. Paris, Éditions Desclée de Brouwer, 1999.

MANEnt Pierre, Histoire intellectuelle du libéralisme, Paris, Calmann-Lévy, 1987.

MARIANO Ricardo, "Le royaume de prospérité de l'Église Universelle », in CORTEN André, Dozon Jean-Pierre, Oro Ari, dirs, Les nouveaux conquérants de la foi: L'Église universelle du royaume de Dieu (Brésil), Paris, Karthala, 2003, p. 197-212.

Marshall-Fratani Ruth, "Prospérité miraculeuse : les pasteurs pentecôtistes et l'argent au Nigéria ", Politique africaine, $\mathrm{n}^{\circ}$ 82, juin 2001, p. 22-44.

Marshall-Fratani Ruth, PÉclard Didier, "Introduction au thème : La religion du sujet en Afrique ", Politique africaine, n 87, octobre 2002, p. 5-19.

MARTIN David, Tongues of Fire: The Explosion of Protestantism in Latin America, Oxford, Blackwell, 1990.

MARy André, " Prophètes pasteurs : la politique de la délivrance en Côte d'Ivoire », Politique africaine, $\mathrm{n}^{\circ} 87$, octobre 2002, p. 69-93.

NiebuHR Richard, The Social Sources of Denominations, Cleveland, World Publishing Co, 1929.

Oro Ari Pedro, "Principios religiosos e práticas políticas de “religiosos políticos” e de “políticos laicos” no Sul do Brasil ", 27e Congrès SISR, Turin 2003.

Poewe Karla, ed., Charismatic Christianity as a Global Culture, Colombia, University of South Carolina Press, 1994.

RANCIÈRE Jacques, La mésentente : politique et philosophie, Paris, Galilée, 1995.

RANCIÈRE Jacques, La haine de la démocratie, Paris, La fabrique éditions, 2005.

RIBEIRO Renato Janine, "Transcendance et immanence dans la politique et le sacré », $27^{e}$ Congrès SISR, Turin 2003.

SANCHIS Pierre, O repto pentecostal à cultura "Catolico-brasileira », Table ronde du Censo evangélica de Rio de Janeiro, ISER, 1992, XXII Reunião Anual da ANPOCS.

Troeltsch Ernst, The Social Teaching of the Christan Churches, New York, Macmillan, 1912.

VAN DijK Rijk, "Time and Transcultural Technologies of the Self in the Ghanaian Pentecostal Diaspora ", in Corten André, Marshall-Fratani Ruth, eds, Between Babel and Pentecost. Transnational Pentecostalism in Africa and Latin America, LondresBloomington, IN, Hurst Publisher/Indiana University Press, 2001, p. 216-234. 
Mots-clés : transcendance et immanence, néo-pentecôtisme, transnationalisation, imaginaire social

\section{Résumé}

Jugé plus transcendant que la religiosité catholique populaire, le pentecôtisme - toutes formes confondues - apparaît aujourd'hui comme immanent en raison des réseaux transnationaux dans lequel ces formes se développent. Pour voir le rapport entre immanence et transnationalisation, le présent texte propose de les traiter au niveau des imaginaires. Considéré ainsi, le rapport paradoxal entre argent et sacré produit des images signifiantes inédites. L'expression "supermarché de la foi », souvent accolée au dit "néo-pentecôtisme", ne peut plus être reçue au premier degré. La transnationalisation s'opère dans le cadre d'imaginaires qui bouleversent les repères de temps et d'espace et s'organisent dans des rituels, des mythes et des icônes se plaçant dans un topos d'horizontalité.

\section{Abstract}

Judged more transcendent than popular catholic religiosity, pentecostalism - in all its forms - appears today as immanent by reason of the transnational networks in which its forms develop. To see the relationship between immanence and transnationalism, this text proposes to treat them at the level of the imaginary. Thus considered, the paradoxical relation between money and the sacred produces novel signifying images. The expression "supermarket of faith", often coupled with so-called "neopentecostalism", cannot be taken at face value. Transnationalisation operates in the framework of the imaginary, which disrupts the markers of time and space and organises through ritual, myth, and icons placed in a horizontal topos.

\section{Resumen}

Juzgado más transcendental que la religiosidad católica popular, el pentecostalismo - en todas sus formas - aparece hoy en día como inmanente en razón de las redes internacionales en las cuales esas formas se desarrollan. Para ver la relación entre inmanencia y transnacionalización, el presente texto propone tratarlos a nivel de los imaginarios. Considerado así, la relación paradójica entre dinero y sagrado produce imágenes significantes inéditas. La expresión "supermercado de la fe ", frecuentemente ligada al llamado "neopentecostalismo ", ya no puede ser recibida en primer grado. La transnacionalización se opera en el cuadro de imaginarios que transtornan las señales de tiempo $y$ de espacio, y que se organizan en rituales, mitos e iconos ubicados en un "topos" de horizontalidad. 